\title{
Perceptual salience of acoustic differences between conspecific and allospecific vocalizations in African collared-doves
}

\author{
GABRIËL J. L. BECKERS, BRIGITTE M. A. GOOSSENS \& CAREL TEN CATE \\ Behavioural Biology, Institute of Evolutionary and Ecological Sciences, Leiden University
}

(Received 17 April 2002; initial acceptance 6 June 2002;

final acceptance 14 August 2002; MS. number: 7296)

\begin{abstract}
In speciation events, species-distinct vocal signals can diverge acoustically in many ways. Signal receivers have to be able to distinguish conspecific from allospecific vocalizations, and the perceptual salience of acoustic features is therefore expected to be an important factor in the evolution of such vocalizations. We tested how dissimilar the species-identifying perch-coos of 12 closely related turtle-dove species (genus Streptopelia) are, as perceived by one of its members, S. roseogrisea. With operant, psychoacoustic methods we trained six doves to respond only to their conspecific coo. Responses to the perch-coos of the 12 other dove species were used as a measure of their perceptual similarity to conspecific perch-coos. Turtle-doves differentiated between the allospecific coos: some were perceived as more similar to their own species' coo than others. With multiple regression analysis we identified three acoustic features that correlated with these differences in perceptual similarity: coo duration, minimum frequency and Wiener entropy. In contrast to findings in other bird species, duration was by far the most important feature in the discrimination between conspecific and allospecific vocalizations for $S$. roseogrisea. The results suggest that this is due not only to the coos of the various species differing in duration but also to a comparatively high perceptibility of the differences in duration.
\end{abstract}

() 2003 Published by Elsevier Science Ltd on behalf of The Association for the Study of Animal Behaviour.

Acoustic signals of closely related bird species are often distinctly different (Becker 1982). This is especially true for long-distance vocalizations that are involved in mate attraction and territorial defence. Species-distinguishing characteristics of such signals are probably important because the use of visual cues is difficult over larger distances, when sender and receiver are often out of view.

During speciation, vocal signals can diverge acoustically in many ways. Changes in, for example, song duration, element ('note') structure, frequency range, frequency modulation, amplitude modulation and tonality of vocalizations may be used to separate the vocalizations of incipient species in signal space. Which parameters are more likely to change than others depends on several factors. One of these is that possibilities for change in the way sound signals are produced may favour particular directions for signal evolution and disfavour others. Limits to the dynamics of vocal tract movements during song production, for example, probably constrain the development and evolution of syllable repetition rate

Correspondence and present address: G. J. L. Beckers, Medical Sciences, Indiana University, 315 Jordan Hall, 1001 East Third Street, Bloomington, IN 47408, U.S.A. (email: gabecker@indiana. edu). B. M. A. Goossens \& C. ten Cate are at Behavioural Biology, Institute of Evolutionary and Ecological Sciences, Leiden University, P.O. Box 9516, 2300 RA Leiden, The Netherlands. in emberizid songbirds (Podos 1997). Properties of the environment through which sound signals are propagated also impose limitations on the acoustic characteristics of vocalizations, if they are to be transmitted efficiently. Higher frequency vocalizations, for example, generally do not carry as far in forested habitat as in open fields (Wiley \& Richards 1982). Whatever the selection pressures or mechanisms of production, however, acoustic changes in vocalizations can be selected for only if they are salient to the receiver. Characteristics of perceptual systems are therefore also expected to be important in signal evolution.

The perceptual salience of acoustic features in vocalizations can be studied only with psychoacoustic experiments in the laboratory, because playback experiments in the field do not allow one to distinguish the salience of features from their functional relevance. When an animal in the field responds differentially to playback vocalizations, one knows that it must have perceived their differences. But when it does not respond, there is no way of knowing whether it did not perceive the differences, or whether it simply was not motivated to respond differentially in that particular context. Furthermore, even when animals do respond differentially, response strength will be determined by the functional relevance of differences rather than their perceptual salience. 
The role of acoustic features in discriminating vocalizations has been studied with psychoacoustic techniques in a number of bird species, for example, budgerigars, Melopsittacus undulatus (Dooling et al. 1987, 1992; Brown et al. 1988), zebra finches, Taeniopygia guttata (Okanoya \& Dooling 1991; Dooling et al. 1992), canaries, Serinus canaria (Dooling et al. 1992), Bengalese finches, Lonchura striata domestica (Okanoya \& Kimura 1993), great tits, Parus major (Weary 1990), red-winged blackbirds, Agelaius phoeniceus (Sinnott 1980), and brown-headed cowbirds, Molothrus ater (Sinnott 1980). None of these studies, however, addressed the perceptual differences in speciesidentifying vocalizations of closely related species, making it difficult to relate the results to an evolutionary context.

We examined the perceptual salience of acoustic differences in vocalizations of closely related species, by using turtle-doves (genus Streptopelia, 17 species, Johnson et al. 2001) as a model system. Turtle-doves produce longdistance vocalizations, called perch-coos (Fig. 1), that are highly stereotypic within species, but distinctly different between species. Slabbekoorn et al. (1999) showed that species identity of individual birds can be correctly established by their perch-coo alone. In contrast, shortdistance coo types (bow- and nest-coos), are not always distinctly different between species (S. R. de Kort \& C. ten Cate, unpublished data). This suggests that turtle-doves use perch-coos to identify conspecific birds. Experimental evidence from playback studies in the field shows that turtle-doves respond differently to conspecific and allospecific perch-coos (de Kort \& ten Cate 2001), so they do indeed discriminate between these signals in the absence of visual cues.

We reported previously on the perceptual relevance of species-specific differences in perch-coos for two turtledove species, $S$. decaocto and S. chinensis (Beckers \& ten Cate 2001). Using an operant design and synthetic coo model stimuli differing only in the parameters of interest, we showed that both species use amplitude modulation structure and temporal structure for acoustic discrimination. The advantage of this experimental design is that we can confirm whether a specific acoustic feature is used for discrimination. On the other hand, it does not allow us to test more than two parameters at the same time. Streptopelia species' perch-coos differ in several acoustic features, such as duration, temporal sound element structure, frequency range, peak frequency, frequency modulation pattern, amplitude modulation (trilled elements) and tonal structure (e.g. Fig. 1). This makes testing the perceptual salience of all these parameters a tedious task. In addition, the required use of artificial stimuli is a drawback, because ultimately we are interested in the perception of real vocalizations.

In the current study, we therefore applied a different approach. We made use of the doves' differential responses to natural conspecific and allospecific perchcoos to investigate the perceptual salience of acoustic differences between species-specific signals. By using psychoacoustic, operant methods we trained domesticated S. roseogrisea to peck a key in response only to conspecific perch-coos. In two methodologically different tests we used the probability of key pecks in response to the perch-coos of 12 other dove species as a measure of their perceptual similarity to conspecific perch-coos. We then used the differential response scores of allospecific coos to identify underlying acoustic variables that correlated with the doves' perception of coo similarity.

\section{METHODS}

\section{Subjects}

We used one female and five male ring doves, a domesticated form of the African collared-dove, S. roseogrisea. Although this domesticated form is sometimes also referred to as 'S. risoria', it is considered to be the same species (Baptista et al. 1997). All birds were obtained commercially, and were experimentally naïve. When not participating in tests, they were housed individually in cages $(80 \times 60 \mathrm{~cm}$ and $60 \mathrm{~cm}$ high), in a room that did not contain any other birds. Each day birds were transferred to an operant test cage for training or testing sessions. To meet with human working schedules, these sessions lasted for three birds from 0900 to 1700 hours, and for the other three from 1700 to 0900 hours. Lights were switched on at 0800 and off at 2000 hours, so the latter group effectively had a session that lasted for $3 \mathrm{~h}$ in the afternoon and $1 \mathrm{~h}$ in the morning. The assignment of individual birds to these session periods was counterbalanced weekly. The birds' diet consisted of a commercially available dry seed mix for turtle-doves, which they could normally obtain only by performing their operant task during the sessions. Birds could initiate trials whenever they were motivated to do so, and hence could regulate their food supply. After each session we measured the amount of food each bird had eaten and, if necessary, gave them a supplementary quantity. Subjects were weighed twice a week to make sure that their body weight did not fall below $90 \%$ of their free-feeding weight. After the experiments were finished, all subjects remained in our laboratory for other behavioural studies. This study was approved by the Animal Experiments Committee of Leiden University.

\section{Operant Apparatus}

We used three identical operant cages, each placed in a separate sound-attenuating chamber, for training and testing procedures. The test cages measured $60 \times 50 \mathrm{~cm}$ and $60 \mathrm{~cm}$ high, and were built of an open wooden frame, the top and three sides of which were covered with wire mesh. The fourth side was of plywood and held the control panel. On the top side, the control panel held a loudspeaker from which sound stimuli were presented. In the centre, two microswitch keys (diameter $1 \mathrm{~cm}$ ) with built-in, red light-emitting diodes (LEDs) were placed $16 \mathrm{~cm}$ apart. The left key functioned as the 'observation key' and the right as the 'report key'. A food dispenser was mounted between the keys. Keys and food dispenser were accessible to the bird via a perch. Illumination was provided by a high-frequency fluorescent light on top of the 


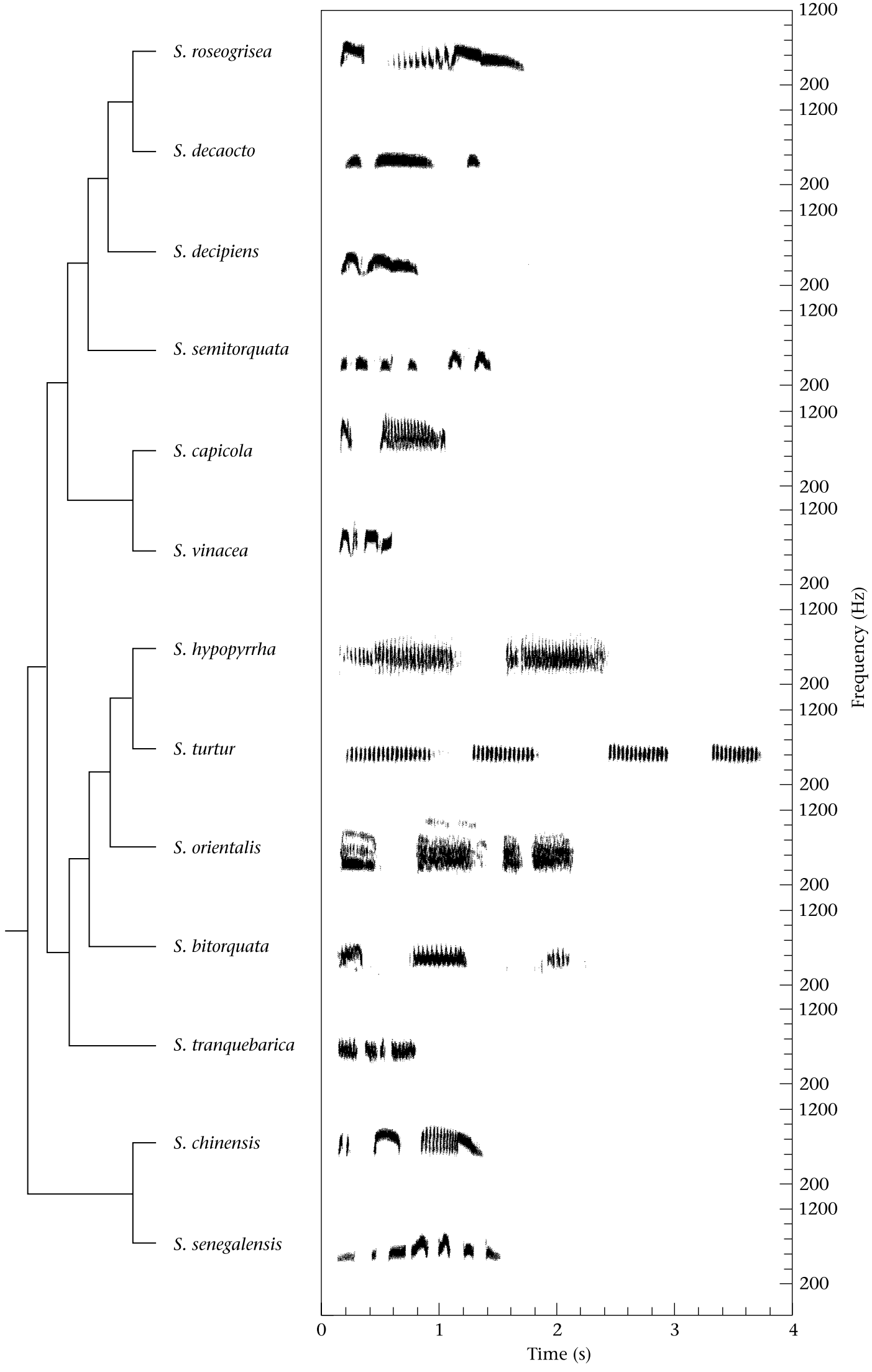

Figure 1. Phylogenetic relationship of 13 Streptopelia species and spectrograms of their perch-coos (Johnson et al. 2001). Branch lengths are not proportional to genetic distance. 
cage. We could observe the birds through one-way screens in the doors of the sound-attenuating chambers. All walls of the sound-attenuating chambers (but not the floor) were completely covered with sound-absorbing foam $(4 \mathrm{~cm}$ thick) to reduce adverse effects from the reflection of sound waves.

Each operant test cage was controlled by a PC and custom-written software. A small, custom-built computer device that was developed at the workshop of our institute functioned as an interface between computer and operant test cage. This device was also used to store digitally and play back sound stimuli.

\section{Stimuli}

We selected six perch-coo vocalizations from different individuals of each of 13 Streptopelia species (Fig. 1). One of these was $S$. roseogrisea, the species to which the test subjects belonged.

We used field-recorded coos if they were available and had a good signal-to-noise ratio. These coos originated from different individuals at sites in Cameroon, Uganda, the Philippines, Great Britain and the Netherlands. Field observations suggest that actively cooing birds, as used for recordings in our case, are almost always males. However, we cannot be sure that all recordings used were from males. For five of the 78 coos we did not have suitable field recordings, so we used recordings from our laboratory (two S.chinensis, one S. orientalis and two S. tranquebarica). Although our subjects were domestic $S$. roseogrisea, we used coo recordings from wild S. roseogrisea to avoid the introduction of systematic, recordingrelated differences between conspecific and allospecific coos in our tests. To the human ear, perch-coos of domestic and wild S. roseogrisea do not sound different, and visual inspection of spectrograms (personal observation) and acoustic analyses (Slabbekoorn et al. 1999) revealed no differences between the coos of the two forms.

Coos were sampled (44.1 kilosamples/s, 16 bit resolution) with a CardDeluxe soundcard (Digital Audio Labs) and CoolEdit software (Syntrillium Software Corporation). Using the computer program Praat (version 3.9.11 for Windows, P. Boersma \& D. Weenink, University of Amsterdam, the Netherlands) we bandpassfiltered all signals to remove sound energy outside the signal's frequency range, which was determined visually for each individual signal in a spectrogram (calculated with a Short Time Fourier Transform, Gaussian window, time resolution $15 \mathrm{~ms}$, frequency resolution $87 \mathrm{~Hz}$, dynamic range $40 \mathrm{~dB}$ ). Filtering was applied in the frequency domain with Praat's built-in filtering function (Hann-like band shape, $50 \mathrm{~Hz}$ smoothing). After filtering, all stimuli were matched for root-mean-square sound amplitude. Digital signals were transferred to the playback device with the CardDeluxe soundcard. The device subsequently resampled (26.8 kilosamples/s) the signal using its OKI MSM6388 sound processor. During the experiments, sound stimuli were generated by the same sound processor, which has a built-in filter to prevent imaging effects, and a Blaupunkt CB 4500 loudspeaker.
We equalized the playback intensities of the devices by setting each of them to $65 \mathrm{~dB}(\mathrm{~A})$ SPL for one of the conspecific stimuli, as measured by the maximum reading of a Cel-231 sound level meter, time weighting $125 \mathrm{~ms}$, at the location of the bird's head during tests.

\section{Shaping and Baseline Training}

We used two operant paradigms to assess how much the different allospecific coos resembled conspecific coos perceptually. Both methods are based on a Go/No-go procedure, in which birds are trained to respond to one category of stimulus, but not to respond to another category (Hulse 1995).

First, the birds were autoshaped to peck the lit observation key for the presentation of one of six conspecific perch-coos, selected at random without replacement by the operating software. The presentation of the coo stimulus was immediately followed by a food reward. In the next stage they had to peck the lit observation key to get a conspecific coo stimulus, and when this sound had finished they had to peck the lit report key to get the food reward. When they performed this task well, key lights were gradually dimmed and finally turned off to prevent the LED light from overshadowing the auditory stimulus (Cynx \& Clark 1998).

After the shaping stage we started baseline training. A peck on the observation key initiated a trial with the random ( $P=0.5$, without replacement) presentation of either a conspecific coo stimulus or a silent period lasting for the duration of a randomly selected conspecific coo stimulus. Pecks at the report key in response to a coo resulted in $3 \mathrm{~s}$ of access to food, but pecks in response to a silent period resulted in $20 \mathrm{~s}$ of time-out, during which the cage light was turned off. Pecks at either key during the presentation of a coo or silent period had no effect. If no peck on the report key had occurred within $2 \mathrm{~s}$ after the coo or silent period had finished, the trial ended and the bird could initiate a new trial by pecking the observation key. When the birds reached a level of 75\% correct responses, reinforcement of trials was reduced to a random $85 \%$, that is $15 \%$ of the correct responses were not followed by a reward and 15\% of the incorrect responses were not followed by a punishment. We considered birds ready for testing when they reached a level of $75 \%$ or more correct trials under this regime in three consecutive sessions.

\section{Test 1}

In the first test, trials were identical to those in training sessions, except that the proportion of unreinforced training trials was reduced to a random 5\% and in the remaining 10\% one of the 72 allospecific coos (from 12 species) was given. Which allospecific stimulus was selected for such a trial was determined by a computer algorithm that chose a random one of the 72 stimuli with equal probability without replacement. These probe stimuli were never reinforced. We predicted that birds would peck in response to probe stimuli more often if 
they were perceptually more similar to the conspecific coos, and less often if they were more dissimilar. We finished a test when the number of probe sounds that had been given amounted to an average of 100 per species, which took on average 35 days. After the tests had finished, we determined the ratio of peck responses to each coo stimulus per individual bird. Using a $G$ test of independence (Sokal \& Rohlf 1995), we determined whether a bird had differentiated significantly between conspecific and allospecific coos. For the birds that showed differentiation, we converted the response ratios to perceptual similarity scores, then tested for the significance of differences between allospecific coos with a mixed-model nested ANOVA, and a Tukey's b post hoc test (Sokal \& Rohlf 1995), in which the 72 test stimuli were nested within the 12 species groups.

\section{Test 2}

Immediately after the first test, all birds were subjected to a second test, which was almost identical to the first, except that probe stimuli (the 72 allospecific perch-coos of the 12 species) now became No-go stimuli. A pecking response to any allospecific coo was punished with timeout and pecking responses to conspecific coos remained rewarded. Thus in contrast to test 1 , the birds now actively had to withhold pecking in response to an allospecific sound to avoid punishment. There were no probe trials and no unreinforced trials. We expected birds to learn this task faster or better for allospecific coos that were perceptually more dissimilar to their conspecific coos. Hence, as in test 1 , the ratio of peck responses to each allospecific coo stimulus was considered a measure of its perceptual similarity to the conspecific coos. We had decided a priori to exclude trial sessions (days) in which the birds achieved fewer than $55 \%$ or more than 95\% correct trials, since these would not be informative with respect to the relative differences between responses to different allospecific coos. Four of the six birds sometimes or frequently had sessions in which the overall discrimination level was lower than the $55 \%$ criterion, so that the proportion of trials excluded per bird varied: $0,0,4,10,46$ and $53 \%$. We ended this experiment after 35 days and calculated the pecking response ratio for each stimulus. Over the whole test period we obtained on average 276 trials per tested species' coo (46 per individual stimulus) per bird.

\section{Transfer Test}

The idea behind training the birds on six conspecific coos instead of one was to minimize the possibility that the conspecific reference was, by accident, atypical for $S$. roseogrisea. Nevertheless, we wanted to make sure that the birds had formed a proper reference of conspecific coos, instead of references to potential oddities in the acoustic features of the six training coos. Therefore we subjected the birds to a 1-day transfer test, immediately after test 2 . The transfer test was almost identical to the sessions in test 2 , with the one difference that all six conspecific perch-coos (Go stimuli) had been replaced with six different conspecific perch-coos. We predicted that if the birds' reference during test 2 had been representative of conspecific coos, then the birds would maintain their normal discrimination level (conspecific versus allospecific) after this replacement. To assess whether discrimination levels were significantly different before and after replacement, we used log-linear analysis (Sokal \& Rohlf 1995) in SPSS for Windows, version 10.1, with the two sessions (before and after replacement), the six birds, the two stimulus categories (conspecific and allospecific coos), and the Go response frequencies as factors. A log-linear model was built through backward elimination of factors and interactions that did not contribute significantly ( $P$ to remove was $<0.05$ ). If in the resulting model the Go response frequencies of the two sound categories were independent of session, we concluded that the replacement of the conspecific coos used during training by a new set of conspecific coos had not resulted in an important change in the birds' discrimination behaviour.

\section{Perceptual Similarity Scores}

Because of the design of our tests we cannot directly use the response difference between a particular allospecific coo and the average conspecific coo as a measure of their perceptual similarity. The overall Go response levels and the level of discrimination varied from bird to bird. This affected absolute response levels and the differences in response levels between conspecific and allospecific coos.

Because we were interested in differences between perceptual similarity of allospecific coos, we standardized the Go responses of the allospecific coos per individual bird with a $z$ transform, so that the mean response became zero and the standard deviation one. These scores are referred to as 'perceptual similarity scores'. A higher score means that a particular allospecific coo is more similar to the conspecific coos.

\section{Identification of Acoustic Correlates}

For all coo stimuli used in the operant tests, we measured the acoustic parameters listed in Table 1. Analyses were carried out with Praat except for the features frequency modulation, spectral continuity and Wiener entropy, which were obtained with the program Sound Analysis 2 (Tchernichovski et al. 2000). Spectrograms in Praat were calculated with a Short Time Fourier Transform with a Gaussian window (time resolution $15 \mathrm{~ms}$, frequency resolution $87 \mathrm{~Hz}$ and dynamic range $40 \mathrm{~dB}$ ). Frequency spectra were calculated by a Fast Fourier Transform of the whole coo waveform, zeropadded up to the nearest radix- 2 number. The rhythm of coo sounds was defined as their intensity envelopes (frame length $25 \mathrm{~ms}$ ), and differences between the rhythm of coos were calculated by cross-correlation. The program Sound Analysis was used with its standard settings, except for the calculation of 'pitch', which was based on the peaks of power spectra. 
Table 1. Acoustic features measured from complete perch-coos

\begin{tabular}{|c|c|}
\hline Acoustic feature & Description \\
\hline Number of elements & Number of separate sound elements \\
\hline Duration & Time between start of first sound element to end of final sound element \\
\hline Total duration elements & Sum of duration of separate sound elements \\
\hline Duty cycle & $\begin{array}{l}\text { Proportion of coo that consists of sound (total duration elements/duration } \\
\text { (oo) }\end{array}$ \\
\hline Rhythm & Intensity envelope over time \\
\hline Minimum frequency & Lowest coo frequency visible in spectrogram \\
\hline Maximum frequency & Highest coo frequency visible in spectrogram \\
\hline Frequency span & Maximum frequency-minimum frequency \\
\hline Peak frequency & Frequency with highest amplitude in frequency spectrum \\
\hline Spectrum bandwidth & Bandwidth of frequency spectrum $20 \mathrm{~dB}$ below peak frequency \\
\hline Lowest frequency of band & Lowest frequency in spectrum at $20 \mathrm{~dB}$ below peak frequency \\
\hline Highest frequency of band & Highest frequency in spectrum at $20 \mathrm{~dB}$ below peak frequency \\
\hline Frequency modulation* & Average slope of frequency change over time \\
\hline Spectral continuity* & Measure of continuity of spectral features over time \\
\hline Wiener entropy* & $\begin{array}{l}\text { Measure of the degree of concentration of sound energy in frequency } \\
\text { spectrum, and thus of randomness of sound wave }\end{array}$ \\
\hline Fraction trill & Proportion of coo that is amplitude modulated \\
\hline Trill rate & Modulation frequency of amplitude-modulated (part of) coo \\
\hline
\end{tabular}

All frequency measures were also determined separately for trilled parts of perch-coos. For details on measurements see text.

*Procedures for calculating these parameters are given in Tchernichovski et al. (2000).

The acoustic measurements of the six conspecific perch-coos were averaged. For each allospecific coo we then calculated for each parameter the difference from the conspecific average. These differences were in turn divided by the conspecific average. Expressing acoustic differences in fractions of the conspecific average value makes it possible to quantify the comparison of different parameters (e.g. peak frequency and duration).

To examine which acoustic features of the allospecific coos can explain the differences in perceptual similarity scores, we used multiple regression analysis (Sokal \& Rohlf 1995), using SPSS for Windows. One of the prerequisites of this type of analysis is that the independent variables entered into the model are not strongly correlated. Therefore we first performed a Pearson correlation test on all combinations of parameters. If a parameter pair had a correlation coefficient of more than 0.7 we did not enter the parameter that had the lowest correlation with perceptual similarity into the regression analysis. Regression models were built with a stepwise method, with the criterion of $P<0.05$ for entering, and $P>0.1$ for the removal of variables. When necessary, we applied a natural logarithmic transform to variables to satisfy the assumptions of regression analysis

\section{RESULTS}

All birds acquired their initial operant task of pecking the report key in response to conspecific coo stimuli and withholding pecks in the absence of such stimuli, at the criterion of $75 \%$ or more correct trials per session. With the introduction of allospecific probe coos in test 1 , however, only four of the six birds showed significantly different pecking responses between the different allospecific coos ( $G$ test: $G_{11}=136.1,118.3,52.3,49.3, P<0.05$;
$G_{11}=20.1,19.1$, NS). Overall, response ratios to allospecific probe coos appeared to be high: an average of 0.81 versus 0.90 for conspecific coos. When allospecific coos became No-go stimuli, in test 2, all birds acquired their new operant task. The Go response ratio to allospecific coos was lower than in test 1: on average 0.67 versus 0.94 for conspecific coos.

\section{Acoustic Differentiation of Allospecific Coos}

Analysis of variance with similarity scores grouped per coo species showed significant differences in perceptual similarity between coos of the 12 species tested (nested ANOVA: test 1: $F_{71,360}=2.26, P<0.001$; test 2 : $F_{71,360}=4.56, P<0.001$; Fig. 2). A correlation test confirmed that the overall results from tests 1 and 2 were similar (Pearson correlation: $r_{10}=0.96, P<0.001$ ).

\section{Acoustic Correlates of Differentiation}

Because the perceptual similarity scores of individual coos in test 2 were based on more operant trials per individual dove (46 versus 17 in test 1 ) and on more individual doves (six versus four in test 1), we used the similarity scores of test 2 as the dependent variable in the multiple regression analysis to identify acoustic correlates of perceptual similarity. We found three acoustic parameters with significant linear regression functions (Table 2): duration, minimum frequency and the natural logarithm of Wiener entropy. A regression model consisting of all three variables was highly significant (ANOVA: $\left.F_{3,68}=81.8, P<0.001\right)$, and explained $78 \%$ of the variance in perceptual similarity scores (Fig. 3).

The acoustic features duration and minimum frequency had significant correlations higher than 0.7 with 
Test 1

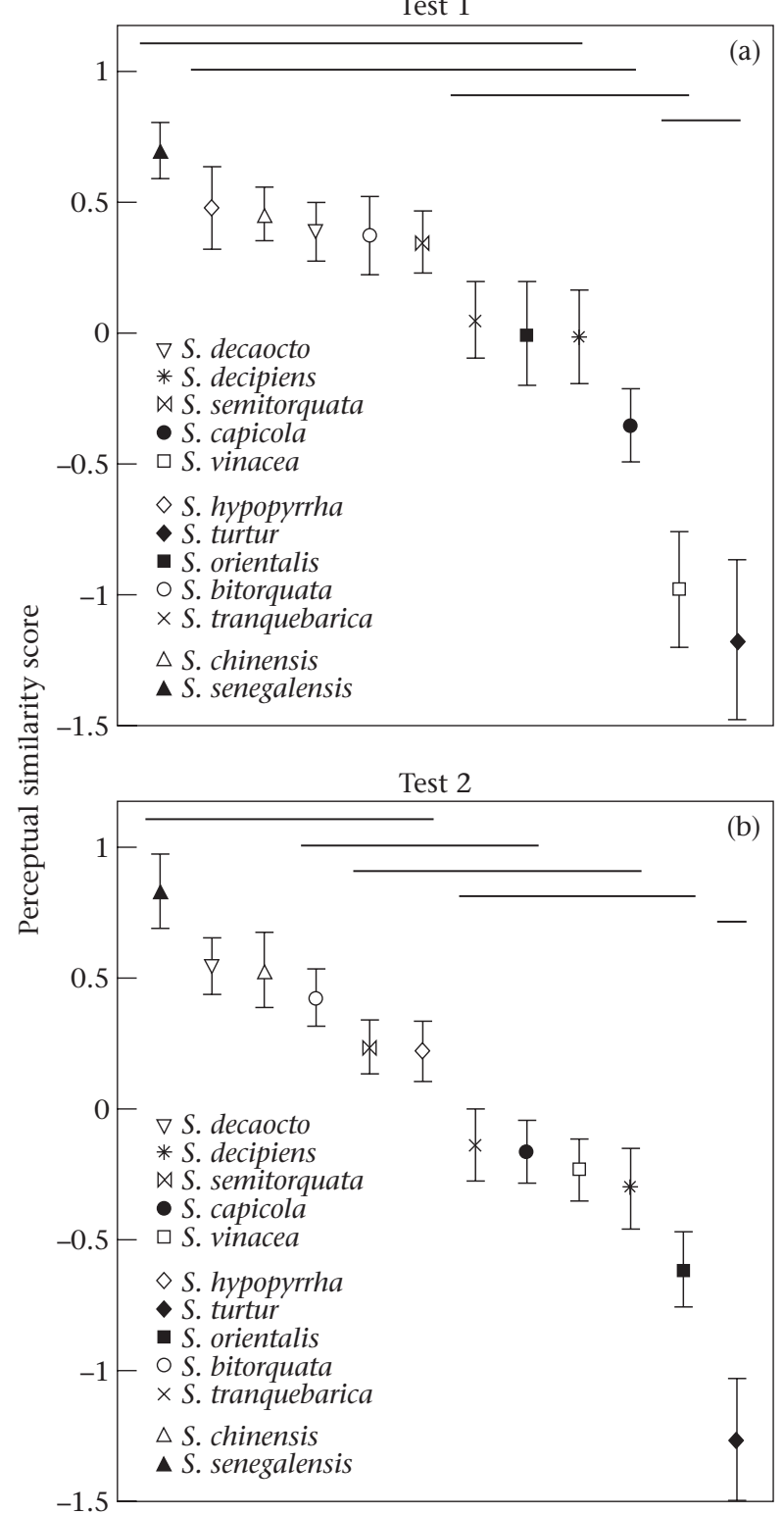

Figure 2. Perceptual similarity scores of allospecific perch-coos, grouped per species of origin. Symbols indicate the mean $\pm S E$ of the mean scores of individual birds. Mean scores of individual birds are based on the perceptual similarity scores of six stimuli per species category. Horizontal lines indicate statistically homogeneous groups (nested ANOVA, Tukey's b post hoc test: $P=0.05$ ).

other parameters (total duration elements, and peak frequency and lowest frequency of band, respectively), which for this reason had not been entered in the regression analysis. Exchanging these parameters and rerunning the analysis resulted in significant models consisting of three parameters, but always with less variance explained.

\section{Transfer Test}

The exchange of the six conspecific coos by six new ones in the transfer test after test 2 did not have a
Table 2. Multiple regression model of acoustic features on perceptual similarity

\begin{tabular}{lccc}
\hline Acoustic feature & $R^{2}$ & $B \pm \mathrm{SE}$ & $P$ \\
\hline Duration & & & \\
Minimum frequency & 0.548 & $-1.493 \pm 0.112$ & $<0.001$ \\
Wiener entropy (In) & 0.081 & $-0.154 \pm 0.031$ & $<0.001$ \\
\hline
\end{tabular}

$R^{2}$ indicates the proportion of the variance of perceptual similarity that is explained by adding that particular feature to the model; $B$ is the partial regression coefficient. $P$ values denote the significance of the partial regression equation.

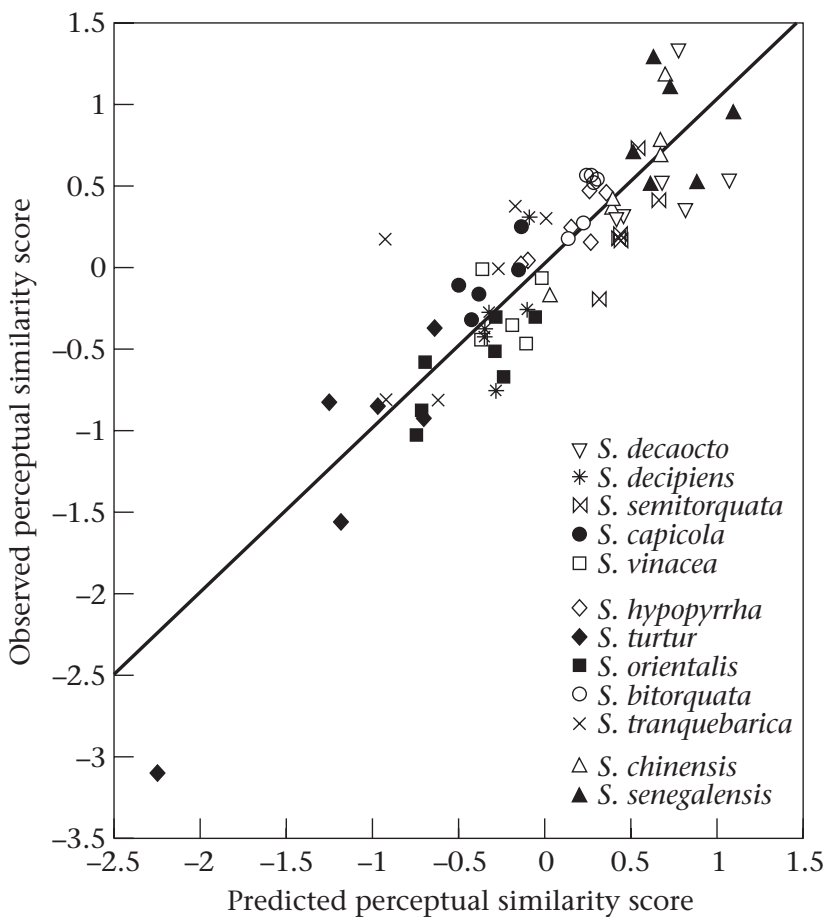

Figure 3. Predictive power of multiple regression model based on the acoustic parameters duration, minimum frequency and natural logarithm of Wiener entropy. The observed perceptual similarity values are standardized peck response ratios ( $z$ transform), and the predicted perceptual similarity values result from the regression model (Table 2). Symbols indicate the similarity score (mean of six subjects) of a particular perch-coo, where different symbols refer to the species of origin. The solid line indicates where predicted values equal observed values.

significant effect on discrimination levels (log-linear analysis: $\chi_{1}^{2}=1.27, P=0.26$ ).

\section{DISCUSSION}

Our results show that $S$. roseogrisea differentiated between allospecific perch-coos when discriminating its own species' perch-coo from that of others. We identified three acoustic features that correlated with this differentiation: coo duration, minimum frequency and Wiener entropy. The overall variance in perceptual similarity due to these features, expressed by the proportion of variance explained in the regression model $\left(R^{2}\right)$, was determined 
by two factors: (1) their perceptibility, expressed by the magnitude of the regression coefficients (B), and (2) the acoustic variance between allospecific coos. Thus, although differences in coo duration could explain 55\% of the observed variance in perceptual similarity scores, and differences in minimum frequency only $15 \%$, the perceptual salience of changes in either parameter was almost equal: the coefficients of their regression functions were -1.5 and -1.4 , respectively. The importance of temporal parameters such as duration in the perceptual differentiation of species' perch-coos fits with earlier observations of Slabbekoorn et al. (1999), who showed with acoustic and statistical analysis that temporal parameters are the most discriminative features between Streptopelia species' perch-coos. The small difference in perceptual salience of changes in a temporal and a frequency parameter, however, is a surprising finding. Just noticeable differences in duration of pure tones are known to be on the order of $10-20 \%$, but only about $1 \%$ for frequency (Dooling 1982). Our results therefore confirm that it is important to distinguish between the perceptual salience of acoustic changes in complex vocalizations and underlying auditory abilities.

The fact that we identified three parameters with significant regression functions does not necessarily mean that these are used perceptually as such. The birds' actual cues may be correlated with the parameters that we measured. Furthermore, we may have missed relevant acoustic features, simply because we did not measure them, or parameters that are correlated with them. On the other hand, the correlates we did find explained $78 \%$ of the observed variance, which leaves only limited room for as yet unidentified features.

The results from the transfer test show that the six conspecific perch-coos that we used in our experiments were representative of conspecific coos in general. Birds maintained their discrimination behaviour between conspecific and allospecific coos after replacement of the conspecific coos used during training by new ones. It is likely that the subjects had formed a perceptual category of these conspecific coos, instead of using each of the six conspecific coos separately as a reference. A less likely explanation that we cannot exclude at present is that the subjects could not tell the difference between the individual conspecific coos, because they are rather stereotypic. For our purposes it does not matter which of these two explanations is correct, because it is clear that whatever the birds referred to, it was not specific to the coo stimuli used in training.

From the finding that duration was the most important parameter in distinguishing conspecific from allospecific perch-coos, it follows that, overall, birds must have listened to the whole stimulus before deciding how to respond. However, it is possible that for very long coos the subjects did not wait for the stimulus to finish before responding. Even worse, they could have given up pecking before the stimulus was finished, which would have been classified as a No-go response. To check for such artefacts, we analysed the response latency times for all sounds in both tests. Mean latency times for individual coos were always at least one standard deviation longer than the coo's duration, that is, the birds waited for the stimulus to finish before responding. This not only rules out the possibility of a response classification artefact but also shows that the birds listened to completed coos before they responded, and were thus able to weight all parameters available.

The outcomes of tests 1 and 2 were very similar, although they originated from methodologically different experiments. This shows that our results are robust with respect to the type of operant task involved. On a species level, the only exceptions seemed to be the coos of S. vinacea and S. orientalis (Fig. 2). Given that both methods gave similar results, we recommend a design similar to our second test (the discrimination design) for future research because of two practical advantages. First, the discrimination design generates many more trials per tested stimulus in a given period, yielding more accurate perceptual similarity scores. Second, the difference between the overall response to conspecific coos and allospecific coos was larger in the discrimination design. Better separation of perceptual scores makes it a more powerful method to identify perceptual differences between vocalizations when they are relatively small.

We previously tested the perceptual relevance of temporal structure ('rhythm') and amplitude modulation structure ('trill') for S. decaocto and S.chinensis doves, when they had to discriminate between synthetic stimuli modelled after their species' perch-coos (Beckers \& ten Cate 2001). The results showed that the birds use both features, with approximately similar weights. In the current study, amplitude modulation structure did not appear to be an important acoustic feature, but one aspect of temporal structure, its duration, did. The stimuli used in our previous experiment differed only in the two parameters of interest. Duration of the separate sound elements and the pauses between them differed markedly, but their total duration did so only slightly: 1.2 and $1.4 \mathrm{~s}$. In our current tests, this would correspond to a difference in perceptual similarity score of less than 0.25 . With only a small difference in duration available, the differences in amplitude modulation apparently became significant cues for the subjects to perform their discrimination task. This illustrates an important point regarding the interpretation of our results: just because a particular parameter was not important in the discrimination tests, this does not mean that birds will not use that parameter when other cues are limited or not available. Overall, the three identified parameters are important when birds discriminate between conspecific and allospecific perch-coos. In specific cases, however, they may not be.

The importance of coo duration in the discrimination of turtle-dove perch-coos contrasts sharply with findings in other bird species. Duration is not important in the discrimination of conspecific from allospecific vocalizations in budgerigars, canaries and zebra finches (Dooling et al. 1992), between allospecific vocalizations in starlings, Sturnus vulgaris (Dooling et al. 1992), and between conspecific vocalizations in budgerigars (Brown et al. 1988). It is likely that at least part of the explanation for the difference between these results and ours is that turtledove perch-coos differ distinctly in temporal structure 


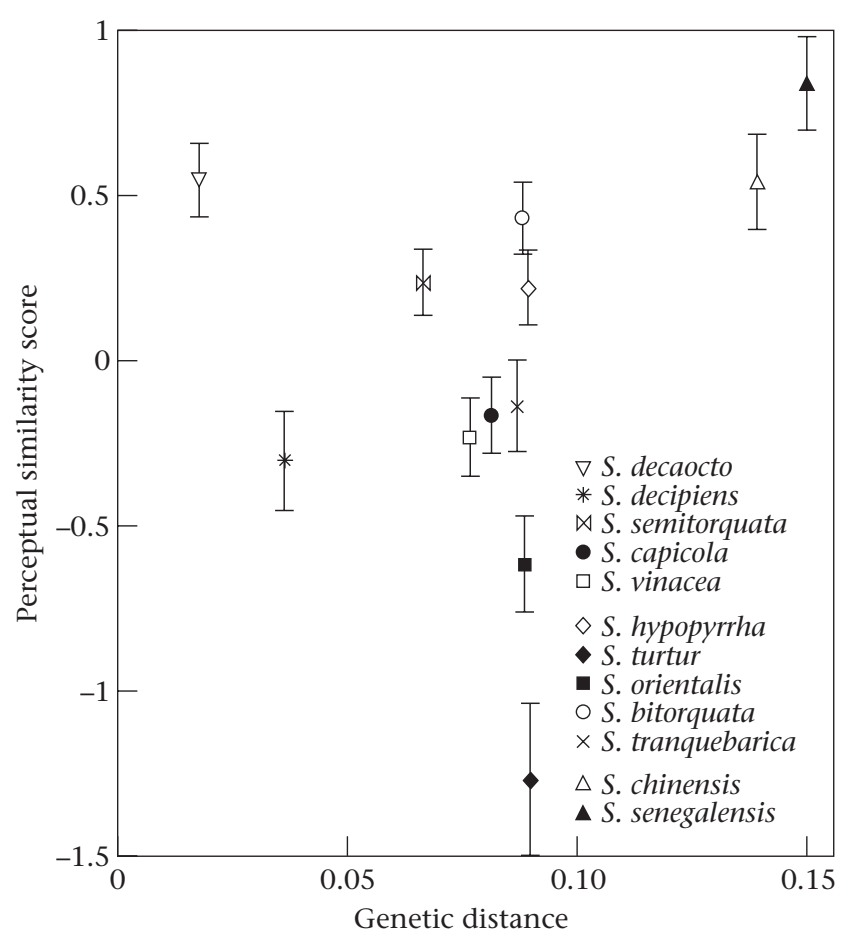

Figure 4. Genetic distance between S. roseogrisea and other species plotted against the perceptual similarity of their perch-coos. Genetic distances are in number of substitutions per site (Johnson et al. 2001), and the perceptual similarity scores are from test 2 in our study.

(Slabbekoorn et al. 1999), of which duration is an important aspect. None the less, the stimulus sets in Dooling et al.'s (1992) study did have significant differences in duration, and yet none of the four species tested used these as a cue for discrimination. This suggests that differences in duration are more salient to turtle-doves than they are to previously tested species. Such an increased sensitivity to temporal differences may have evolved in response to the perch-coos differing predominantly in temporal features. Our methodological approach allows us to separate the effect of perceptibility of differences in acoustic features (regression coefficients) from that of their variance in the stimuli presented. The studies cited above, however, focused on correlations between acoustic features and perceptual differences, and did not distinguish between feature perceptibility and variance. Consequently, a more quantitative comparison between species with respect to the perceptibility of differences in duration and other parameters is not possible at present.

A well-resolved phylogeny recently became available for almost all species in the genus Streptopelia (Johnson et al. 2001), which enables us to examine the perceptual similarity of vocalizations of these species in a phylogenetic context. In Fig. 4 we plotted the genetic distance between $S$. roseogrisea and the other species as reported by Johnson et al. (2001) against the perceptual similarity scores of their perch-coos as obtained in our study. The perceptually most similar coo is from the least related species, S. senegalensis; the sister species of S. roseogrisea,
S. decaocto, also has a high similarity score. From other comparisons it is also noticeable that whether an allospecific vocalization is perceived as similar to the own species' coo is not apparent from its position in the phylogenetic tree. Thus, although turtle-doves do not learn their song (Nottebohm \& Nottebohm 1971), and hence an increased rate of evolutionary change of vocalizations through cultural transmission of mutations, as in songbirds, is not possible, evolutionary differentiation of perch-coos occurred fast enough to obscure a phylogenetic pattern in perceptual similarity. It seems likely that such rapid differentiation is partly due to the perceptually most salient acoustic feature, coo duration, being relatively easy to vary during sound production (Gaunt 1986).

As a more general point, this study shows that the impact of various vocal parameters on signal recognition cannot be deduced directly either from between-signal variation or from psychophysical experiments concentrating on specific parameters. Establishing perceptual salience of various parameters in natural signals as done in the present study is thus essential for interpreting the perceptual relevance of species differences in signal structure.

\section{Acknowledgments}

We thank Selvino de Kort, Rob Lachlan, Albertine Leitão and Nienke Terpstra for commenting on the manuscript.

\section{References}

Baptista, L. F., Trail, P. W. \& Horblit, H. M. 1997. Family Columbidae (pigeons and doves). In: Handbook of the Birds of the World: Sandgrouse to Cuckoos. Vol. 4 (Ed. by J. del Hoyo, A. Elliot \& J. Sargatal), pp. 60-243. Barcelona: Lynx Edicions.

Becker, P. H. 1982. The coding of species-specific characteristics in bird sounds. In: Acoustic Communication in Birds. Vol. 1 (Ed. by D. E. Kroodsma \& E. H. Miller), pp. 213-252. New York: Academic Press.

Beckers, G. J. L. \& ten Cate, C. 2001. Perceptual relevance of species-specific differences in acoustic signal structure in Streptopelia doves. Animal Behaviour, 62, 511-518.

Brown, S. D., Dooling, R. J. \& O'Grady, K. 1988. Perceptual organization of acoustic stimuli by budgerigars (Melopsittacus undulatus): III. Contact calls. Journal of Comparative Psychology, 102, 236-247.

Cynx, J. \& Clark, S. 1998. The laboratory use of conditional and natural responses in the study of avian auditory perception. In: Animal Acoustic Communication (Ed. by S. L. Hopp, M. J. Owren \& C. S. Evans), pp. 353-377. Berlin: Springer-Verlag.

Dooling, R. J. 1982. Auditory perception in birds. In: Acoustic Communication in Birds. Vol. 1 (Ed. by D. E. Kroodsma \& E. H. Miller), pp. 95-130. New York: Academic Press.

Dooling, R. J., Park, T. J., Brown, S. D., Okanoya, K. \& Soli, S. D. 1987. Perceptual organization of acoustic stimuli by budgerigars (Melopsittacus undulatus): II. Vocal signals. Journal of Comparative Psychology, 101, 367-381.

Dooling, R. J., Brown, S. D., Klump, G. M. \& Okanoya, K. 1992. Auditory perception of conspecific and heterospecific vocalizations in birds: evidence for special processes. Journal of Comparative Psychology, 106, 20-28. 
Gaunt, A. S. 1986. Interaction of syringeal structure and airflow in avian phonation. In: XIX Congressus Internationalis Ornithologici (Ed. by H. Ouellet), pp. 913-924. Ottawa: University of Ottawa Press.

Hulse, S. H. 1995. The discrimination-transfer procedure for studying auditory perception and perceptual invariance in animals. In: Methods in Comparative Psychoacoustics. Vol. 6 (Ed. by G. Klump, R. Dooling, R. Fay \& W. Stebbins), pp. 319-330. Basel: Birkhäuser Verlag.

Johnson, K. P., de Kort, S., Dinwoodey, K., Mateman, A. C., ten Cate, C., Lessells, C. M. \& Clayton, D. H. 2001. A molecular phylogeny of the dove genus Streptopelia. Auk, 118, 874-887.

de Kort, S. R. \& ten Cate, C. 2001. Response to interspecific vocalizations is affected by degree of phylogenetic relatedness in Streptopelia doves. Animal Behaviour, 61, 239-247.

Nottebohm, F. \& Nottebohm, M. E. 1971. Vocalizations and breeding behavior of surgically deafened ring doves, Streptopelia risoria. Animal Behaviour, 19, 313-327.

Okanoya, K. \& Dooling, R. J. 1991. Perception of distance calls by budgerigars (Melopsittacus undulatus) and zebra finches (Poephila guttata): assessing species-specific advantages. Journal of Comparative Psychology, 105, 60-72.

Okanoya, K. \& Kimura, T. 1993. Acoustical and perceptual structures of sexually dimorphic distance calls in Bengalese finches
(Lonchura striata domestica). Journal of Comparative Psychology, 107, 386-394.

Podos, J. 1997. A performance constraint on the evolution of trilled vocalizations in a songbird family (Passeriformes: Emberizidae). Evolution, 51, 537-551.

Sinnott, J. M. 1980. Species-specific coding in bird song. Journal of the Acoustical Society of America, 68, 494-497.

Slabbekoorn, H., de Kort, S. \& ten Cate, C. 1999. Comparative analysis of perch-coo vocalizations in Streptopelia doves. Auk, 116, 737-748.

Sokal, R. R. \& Rohlf, F. J. 1995. Biometry: the Principles and Practice of Statistics in Biological Research. 3rd edn. New York: W. H. Freeman.

Tchernichovski, O., Nottebohm, F., Ho, C. E., Pesaran, B. \& Mitra, P. P. 2000. A procedure for an automated measurement of song similarity. Animal Behaviour, 59, 1167-1176.

Weary, D. M. 1990. Categorization of song notes in great tits: which acoustic features are used and why? Animal Behaviour, 39, 450 457.

Wiley, R. H. \& Richards, D. G. 1982. Adaptation for acoustic communication in birds: sound transmission and signal detection. In: Acoustic Communication in Birds. Vol. 1 (Ed. by D. E. Kroodsma \& E. H. Miller), pp. 131-181. New York: Academic Press. 